\title{
THE CONTROLLED-DRIFT DETECTOR: CHARACTERISATION OF THE READOUT MECHANISM AND OF THE CHARGE HANDLING CAPABILITY*
}

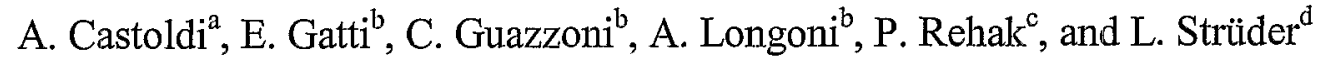 \\ ${ }^{a}$ Politecnico di Milano, Dipartimento di Ingegneria Nucleare, Milano, Italy \\ ${ }^{b}$ Politecnico di Milano, Dipartimento di Elettronica e Informazione, Milano, Italy \\ ${ }^{\mathrm{c} B}$ Brookhaven National Laboratory, Upton, NY 11973 \\ ${ }^{\mathrm{d}}$ MPI Halbleiterlabor, Munich, Germany
}

November, 1998

${ }^{*}$ Work supported in part by the U.S. Department of Energy Contract No. DE-AC02-98CH10886. 


\title{
The Controlled-Drift Detector: Characterisation of the Readout Mechanism and of the Charge Handling Capability
}

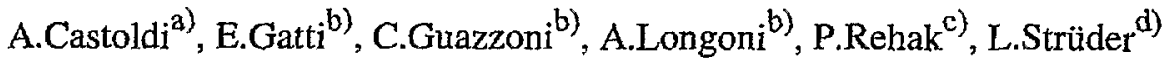 \\ a) Politecnico di Milano, Dipartimento di Ingegneria Nucleare Ce.S.N.E.F., P.za L. da Vinci 32, 20133 Milano, Italy \\ b) Politecnico di Milano, Dipartimento di Elettronica e Informazione, P.za L. da Vinci 32, 20133 Milano, Italy \\ c) Brookhaven National Laboratory, Upton, NY 11973, USA \\ d) MPI Halbleiterlabor, Paul-Gerhardt-Allee 42, D-81245 Miinchen, Germany
}

\begin{abstract}
A new position-sensing $X$-ray detectot operated in integrate-readout mode has been recently designed and characterised. Due to the peculiar working principle of the new detector, the charge handling capability, that is the maximum charge that can be stored in a pixel, is related to the operating conditions of the device. In particular the amplitude of the barriers that confine the signal electrons during the integration phase depends on the applied drift field and on the field perturbation superposed to it. A detailed experimental characterisation of the charge handling capability as a function of these parameters has been carried out.
\end{abstract}

\section{INTRODUCTION}

A new position-sensing $X$-ray detector, called ControlledDrift Detector ${ }^{1}$ (CDD)[1], has been recently introduced. The device is operated in integrate-readout mode. During the readout phase a nearly uniform electrostatic field transports the integrated signal electrons to the output electrode. The drift time gives the position of incidence. During the integration phase the drift is blocked by means of a suitable field perturbation superposed to the drift field. The signal electrons generated by the incident $\mathrm{X}$-ray are collected from the entire volume into an array of potential wells placed at several microns from the front side of the detector.

The n-type bulk (280 $\mathrm{km}$ thick $3 \mathrm{k} \Omega \mathrm{cm}$ tesistivity substrate and $15 \mu \mathrm{m}$ thick $50 \Omega \mathrm{cm}$ resistivity epitaxial layer) is fully depleted by means of the $\mathrm{p}^{+}$field strips implanted on the front side and by a uniform $\mathrm{p}^{+}$implant on the back side.

Figure 1 shows a scheme of principle of the $\mathrm{p}^{+}$field strips of the front side of the detector and of the biasing circuitry. In this design implementation $[2,3]$ the electrostatic field configurations for the integration and the readout phases are generated by a proper biasing scheme of the three resistive dividers, shown in Figure 1. The field perturbation required to block the drift of the signal electrons during the integration phase is generated by the proper choice of the bias change $\Delta V$. The confinement along the other transverse direction is

${ }^{1}$ Patent applications: n. MI 97 A 000423 (Italy, Feb. 27-th, 1997), n. 98830089.3 (European Countries, Feb. 24-th, 1998), n. 09/032130 (U.S.A., Feb. 27-th, 1998) achieved by means of channel-stop deep p-implants [4]. The pixel size is $180 \times 180 \mu \mathrm{m}^{2}$.

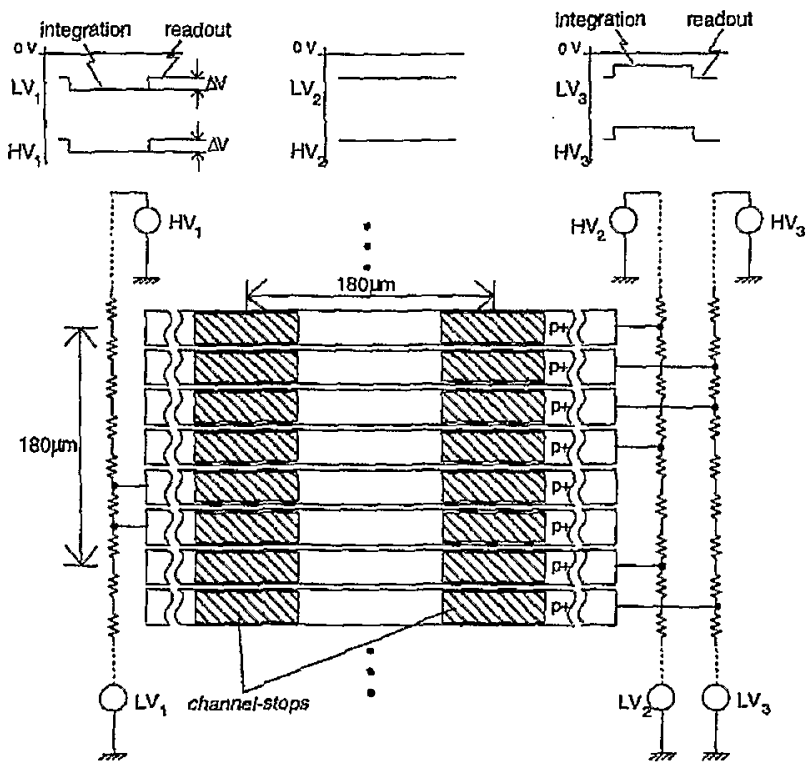

Figure 1: Scheme of principle of the $\mathrm{p}^{+}$field strips of the front side of the detector and of the biasing circuitry.

At a given drift field the bias change $\Delta V$ must be tuned in order to generate a field perturbation able to confine the electrons generated by the $\mathrm{X}$-ray. However as the applied drift field rises, the same field perturbation produces lower confining barriers at the depth where the electrons are stored. Such depth must be traded with the requirement of high uniformity of the drift field during the readout phase that improves with the depth from the surface. Objective of the present work is to test experimentally the charge handling capability as a function of the amplitude of the field perturbation and of the applied drift field. Section $2 a$ reviews the readout mechanism of the CDD together with the experimental measurements carried out to characterise the integrate-readout cycle for different drift fields. Section $2 b$ is devoted to the experimental characterisation of the charge handIing capability of the CDD as a function of the amplitude of the drift field perturbation and of the applied drift field. 


\section{EXPERIMENTAL CHARACTERISATION}

\section{A. Readout mechanism}

The fast readout of the integrated signal electrons achievable with a static drift field has been experimentally verified. The bias change $(\Delta V=2 V)$ needed for the transition between the two operating phases is provided at low level of impedance by connecting external dividers of $2 \mu \mathrm{F}$ capacitors in parallel to two of the on-chip resistive dividers ( 1 node every six) while $1 \mathrm{nF}$ capacitors bypass to ground the intermediate nodes of the third on-chip resistive divider.

A $904 \mathrm{~nm}$ pulsed laser with a pulse duration of few nanoseconds has been focused by means of the optics of a microscope on the front surface of the detector. The laser spot diameter is about $20 \mu \mathrm{m}$ as can be obtained by de-convolution of the experimental data from the metalization pattern of the field strips. The laser intensity was adjusted to generate about 24,000 electrons, corresponding to an X-ray of about $86 \mathrm{keV}$. The light spots were spaced of $30 \mu \mathrm{m}$ along the drift direction. The proper operation of the detector has been tested for drift fields in the range 100-400 V/cm. Figure 2 shows the measured drift time normalised to the drift time from the last pixel versus the drift coordinate. The time axis is divided in equal time bins corresponding to the average drift time between adjacent pixels.

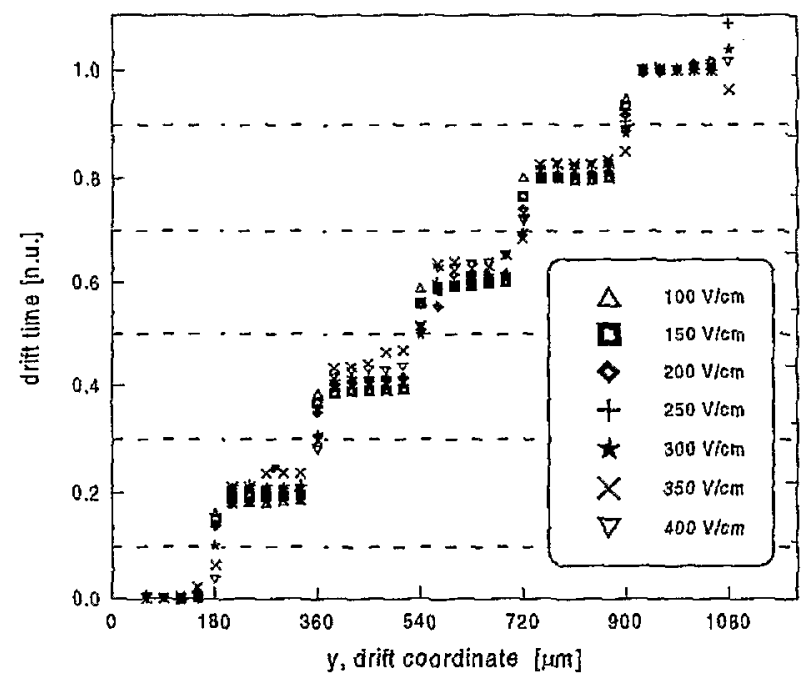

Figure 2: Measured drift time normalised to the drift time from the last pixel versus the drift coordinate for drift fields in the range 100 $400 \mathrm{~V} / \mathrm{cm}$.

The nearly abrupt change in the measured drift time occurs when the laser spot is focused on the saddle point that defines the border between two adjacent pixels of the same column. For all the experimented drift fields the non-linearity of the drift time vs. incidence position curves allows to safely identify the starting pixel. At $300 \mathrm{~V} / \mathrm{cm}$ the measured non- linearity is equal to $1 \%$. The corresponding readout speed of the charge packet is $0.35 \mathrm{~cm} / \mu \mathrm{s}$.

\section{$B$. Charge handling capability}

The amplitude of the potential barriers that block the drift of the signal electrons can be tuned by varying the bias change $\Delta V$ applied to the field strips of the front side.

The fraction of the injected charge that can be confined in the pixel with the device biased at different drift fields in the range $250-400 \mathrm{~V} / \mathrm{cm}$ has been tested as a function of bias change $\Delta \mathrm{V}$ for different levels of charge injection (up to 80,000 electrons). The charge was generated by an IR pulsed laser, focused on the strip overneath the collecting minimum of the first pixel. The time duration of the integration phase was limited to $50 \mu \mathrm{s}$ in order to make the contribution due to the integration of the thermally generated leakage current negligible. In all the operating conditions the signal charge was not leaking over the lateral barriers.

In Figure 3a the charge stored in the pixel, $\mathrm{Q}_{\mathrm{pix}}$ (that is the charge released by the pixel after the transition to the readout phase) notmalised to the total injected charge, $Q_{\text {tot }}=20,000$ electrons, is plotted versus the amplitude of the bias change $\Delta V$ of the strips of the front side of the detector (lower $\mathrm{X}$-axis) for different values of the applied drift field. The upper $x$-axis shows the corresponding amplitudes of the field perturbation at the depth where the electrons are stored $(z \cong 12 \mu \mathrm{m})$.

For small values of the drift field perturbation the generated barriers are not high enough to fully confine all the generated charge. A fraction of the injected electrons is "instantaneously" lost and causes a signal synchronous with the laser trigger to appear at the output electrode. Therefore the charge stored in the pixel $\left(\mathrm{Q}_{\mathrm{pix}}\right)$, proportional to the output signal measured at the anode after the transition to the readout phase, is lower than the total injected charge and can be assumed as an estimation of the charge handling capability of the pixel for such amplitude of the drift field perturbation. For higher values of the field perturbation the barriers are high enough to fully confine all the injected charge $Q_{\text {tor }}$. No output signal synchronous with the laser trigger is present and the charge stored in the pixel $\left(\mathrm{Q}_{\mathrm{pix}}\right)$ is equal to the total injected charge.

The same measurements were repeated for different values of the total injected charge, $Q_{\text {tot }}=40,000$ electrons, $Q_{t 0 t}=60,000$ electrons, $Q_{t o t}=80,000$ electrons and the results are shown in Figures 3b, 3c, 3d.

The knees of the curves define the minimum field perturbation required to confine the injected charge, $Q_{\text {tot }}$. Such value is always greater than the applied drift field. For low levels of charge injection, when the electrostatic repulsion can be neglected, a field perturbation slightly higher than the applied drift field is enough to achieve charge confinement. For a drift field of $250 \mathrm{~V} / \mathrm{cm}$ a drift perturbation of about $300 \mathrm{~V} / \mathrm{cm}$ is enough to fully confine the total injected charge 
$Q_{\text {tot }}=20,000$ electrons. For higher levels of charge injection the amplitude of the field perturbation needed to fully confine the injected charge tends to be greater as higher potential barriers are required to oppose to the electrostatic repulsion.

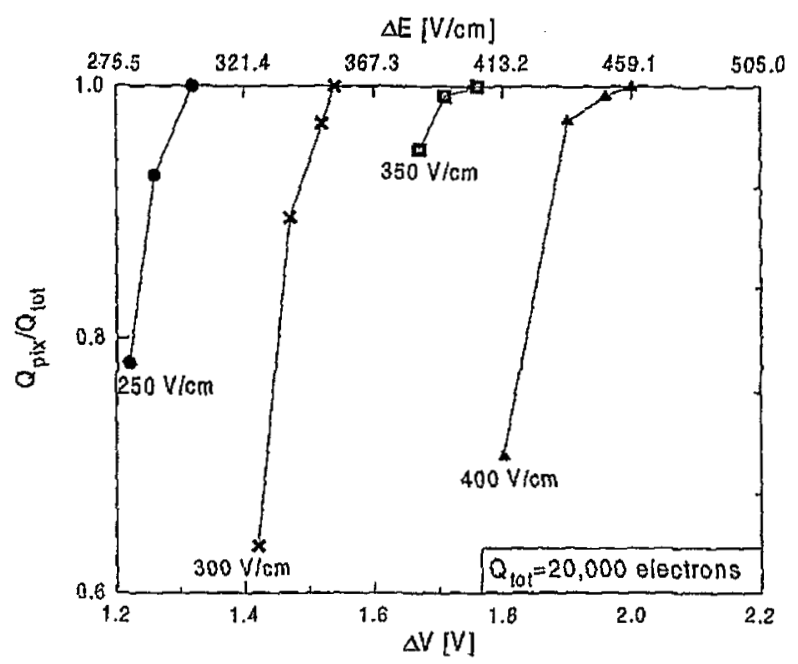

a)

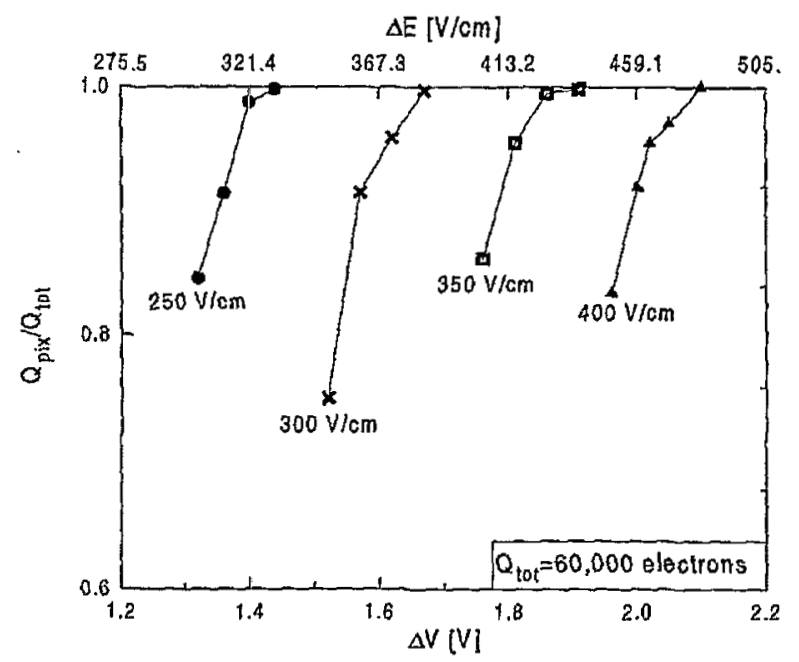

c)

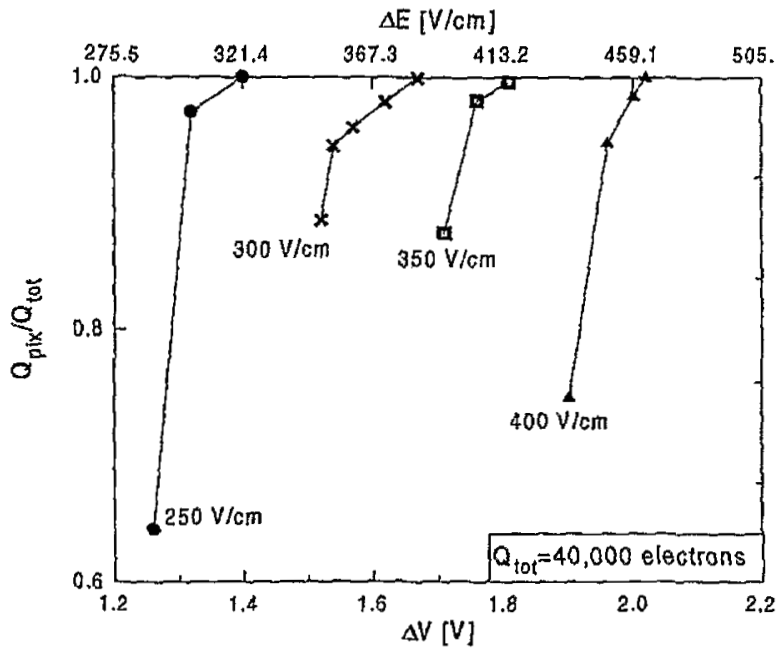

b)

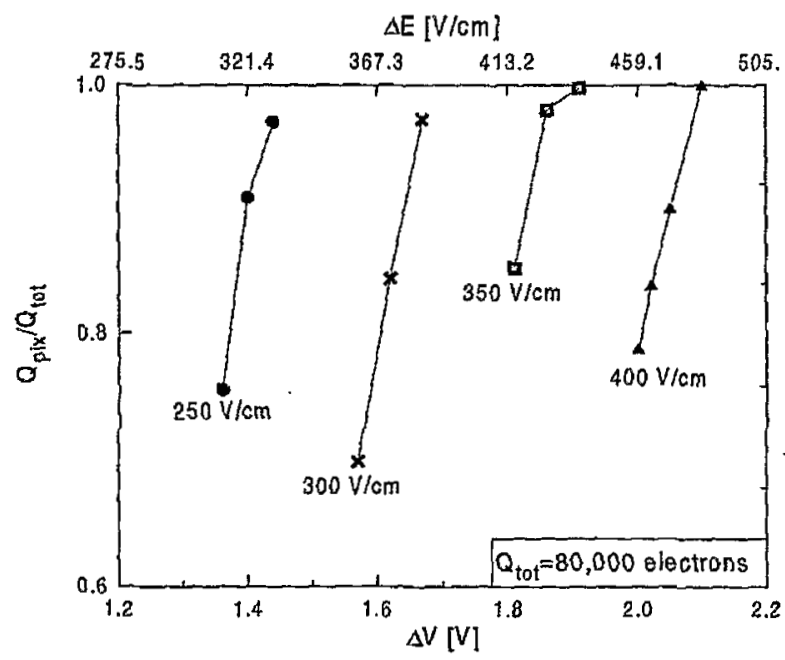

d)

Figure 3: Charge stored in the pixel $\left(Q_{\text {pix }}\right)$ notmalised to the total injected charge $\left(Q_{\text {tot }}\right)$ as a function of the amplitude of the applied perturbation for different drift fields. The lower $\mathrm{X}$-axis shows the bias change $\Delta V$ applied to the field strips of the front side of the detector. The upper $x$-axis shows the corresponding values of the field perturbation $\Delta \mathrm{E}$ at the clepth where the electrons are stored ( $z=12 \mu \mathrm{m}$ ).

a) $Q_{\text {tot }}=20,000$ electrons, b) $Q_{\text {tot }}=40,000$ electrons, c) $Q_{\text {tot }}=60,000$ electrons, d) $Q_{\text {tot }}=80,000$ electrons.

\section{ACKNOWLEDGEMENTS}

E.Gatti and A.Castoldi wish to thank Instrumentation division, BNL for the kind hospitality.

This work was supported by the Italian INFN and by the US Department of Energy under contract number DE-AC0298CH10886. Accordingly, the U.S. Government retains a nonexclusive, royalty free license to publish or reproduce the published form of this contribution, or allow others to do so, for U.S. Government purposes.

\section{REFERENCES}

[1] A.Castoldi, C.Guazzoni, E.Gatti, A.Longoni, P.Rehak, L.Strüder, "Conception and Design Criteria of a Novel Silicon Device for the Measurement of Position and 
Energy of X-Rays," IEEE Trans. Nucl. Science, vol.44, pp. 1724-1732, Oct. 1997.

[2] A.Castoldi, C.Guazzoni, "A New Position Sensing X-Ray Detector with Spectroscopic Capability: Working Principle and Experimental Results," in press on $I E E E$ Trans. Electron Devices.
[3] A.Castoldi, E.Gatti, C.Guazzoni, A.Longoni, P.Rehak, L.Strüder, "The Controlled-Drift Detector," presented at 8th European Symposium on Semiconductor Detectors (in press Nucl. Instr. and Meth., 1999).

[4] A.Castoldi, P.Rehak, P.Foll, "A New Silicon Drift Detector with Reduced Lateral Diffusion,", Nucl. Instr. and Meth., vol. A377, pp.375-380, August 1996. 